\title{
Of Own and Forced Vibrations of Dissipative Inhomogeneous Mechanical Systems
}

\author{
I. I. Safarov, M. Kh. Teshayev, B. Z. Nuriddinov, Z. I. Boltayev \\ Buchara Technological-Engineering Institute, Bukhara City, Uzbekistan \\ Email: muhsin_5@mail.ru
}

How to cite this paper: Safarov, I.I., Teshayev, M.Kh., Nuriddinov, B.Z. and Boltayev, Z.I. (2017) Of Own and Forced Vibrations of Dissipative Inhomogeneous $\mathrm{Me}$ chanical Systems. Applied Mathematics, 8, 1001-1015.

https://doi.org/10.4236/am.2017.87078

Received: June 2, 2017

Accepted: July 24, 2017

Published: July 27, 2017

Copyright () 2017 by authors and Scientific Research Publishing Inc. This work is licensed under the Creative Commons Attribution International License (CC BY 4.0).

http://creativecommons.org/licenses/by/4.0/

\begin{abstract}
In the work, the problems of proper and forced oscillations of dissipative mechanical systems, consisting of rigid and deformable bodies are solved. To quantify the dissipative properties of the system, two values are proposed: the minimum resonance frequency of natural oscillations and the maximum resonant amplitude. In the study of the problem of dissipative inhomogeneous mechanical systems, a nonmonotonic dependence of the damping coefficients on the parameters of the system was observed. The concepts are derived Global damping factor, which characterizes the Damping properties of the dissipative mechanical system as a whole.
\end{abstract}

\section{Keywords}

Own Waves, Dissipative Body, Environment, Spectral Problem, Natural Frequency, Phase Velocity

\section{Introduction}

The use of damping vibrations of dynamic viscoelastic mechanical systems with different rheological properties, for all the studies of the problem [1] [2], is rarely considered in the scientific literature. At the same time, modern machine building is characterized by a wide use of polymeric and metallic materials with various viscoelastic properties [3] [4] [5] [6]. A mechanical system, consisting of rigid and deformable bodies, connected to each other and to a base by deformable (elastic or viscoelastic) elements, is studied.

\section{Statement of the Problem and Methods of Solution}

Let us consider a composite structure occupying a volume $V=\sum_{n=1}^{N} V_{n}$, bounded by a surface $\sum=\sum_{u}+\sum_{p}$. Each of $N$ volumes $V_{n}$ is filled with a viscoelastic 
medium, the parameters of which depend on the number $n$. On a part of the surface $\Sigma_{u}$-zero motions, on $\Sigma_{p}$-zero stresses; mass forces are absent. The frequencies and damping indices of the natural vibrations of the structures are to be determined. The physical properties of the material of the $n^{\text {th }}$ volume of the structure, are described by the relations:

$$
\sigma_{i j}=\tilde{\lambda}_{n} \varepsilon_{k k} \delta_{i j}+2 \tilde{\mu}_{n} \varepsilon_{i j}(i, j, k=1,2,3 ; n=1,2, \cdots, N),
$$

where $\sigma_{i j}, \varepsilon_{i j}$-components of stress and strain tensors , $\tilde{\lambda}_{n}, \tilde{\mu}_{n}-$ Volterra operators

$$
\begin{aligned}
& \tilde{\lambda}_{n} \varphi(t)=\lambda_{n}\left[\varphi(t)-\int_{0}^{t} R_{\lambda n}(t-\tau) \varphi(\tau) \mathrm{d} \tau\right] ; \\
& \tilde{\mu}_{n} \varphi(t)=\mu_{n}\left[\varphi(t)-\int_{0}^{t} R_{\mu n}(t-\tau) \varphi(\tau) \mathrm{d} \tau\right]
\end{aligned}
$$

here $\lambda_{n}, \mu_{n}, R_{\lambda n}, R_{\mu n}$-Lame parameters and the relaxation core of the medium occupying the volume $V_{n} ; \varphi(t)$-an arbitrary function of time.

We assume, that the integral terms in (1) are small. Let the functions $\varphi(t)$ has the form $\varphi(t)=\psi(t) \mathrm{e}^{-i \omega_{R} t}$, where $\psi(t)$-slowly varying function of time, $\omega_{R}$-real constants. Further, applying the freezing procedure, which has been widely studied [7], to the place (1), we can obtain approximate relations:

$$
\begin{aligned}
& \tilde{\lambda}_{n} \varphi \approx \bar{\lambda}_{n} \varphi=\lambda_{n}\left[1-\Gamma_{\lambda n}^{C}\left(\omega_{R}\right)-i \Gamma_{\lambda n}^{S}\left(\omega_{R}\right)\right] \varphi ; \\
& \tilde{\mu}_{n} \varphi \approx \bar{\mu}_{n} \varphi=\mu_{n}\left[1-\Gamma_{\mu n}^{C}\left(\omega_{R}\right)-i \Gamma_{\mu n}^{S}\left(\omega_{R}\right)\right] \varphi ; \\
& \Gamma_{\lambda n}^{c}\left(\omega_{R}\right)=\int_{0}^{\infty} R_{\lambda n}(\tau) \cos \omega_{R} \tau \mathrm{d} \tau ; \Gamma_{\mu n}^{c}\left(\omega_{R}\right)=\int_{0}^{\infty} R_{\mu n}(\tau) \cos \omega_{R} \tau \mathrm{d} \tau ; \\
& \Gamma_{\lambda n}^{s}\left(\omega_{R}\right)=\int_{0}^{\infty} R_{\lambda n}(\tau) \sin \omega_{R} \tau \mathrm{d} \tau ; \Gamma_{\mu n}^{s}\left(\omega_{R}\right)=\int_{0}^{\infty} R_{\mu n}(\tau) \sin \omega_{R} \tau \mathrm{d} \tau .
\end{aligned}
$$

Physical relationships for deformable elements of zero volume

$$
F_{e}=-\bar{C}_{e} \Delta l=-C_{e}\left[1-\Gamma_{e}^{C}\left(\omega_{R}\right)-i \Gamma_{e}^{S}\left(\omega_{R}\right)\right] \Delta l,
$$

where $\Delta l$-lengthening this element; $C_{e}$-instant stiffness of the massless element. When posing the problem of proper and forced oscillations of a dissipative mechanical system, consisting of rigid and deformable bodies, the principle of possible displacements is used, according to which the sum of all active forces, acting on the system, including inertia forces, is zero:

$$
\delta A=\delta A_{F}+\delta A_{u}+\delta A_{I}=0 .
$$

where,

$$
\begin{aligned}
& \delta A_{F}=-\sum_{n=1}^{s_{2}} \int_{V_{n}} \sigma_{i j} \delta \varepsilon_{i j} \mathrm{~d} V-\sum_{e=1}^{s_{1}} F_{e} \Delta l ; \\
& \delta A_{u}=-\sum_{n=1}^{s_{2}} \int_{V_{n}} \rho_{u} \frac{\partial^{2} \mathbf{u}}{\partial t^{2}} \delta \boldsymbol{u} \mathrm{d} V-\sum_{k=1}^{n} m_{k} \frac{\mathrm{d}^{2} \boldsymbol{u}}{\mathrm{d} t^{2}} \delta \mathbf{u}_{k}-\sum_{k=1}^{n} I_{k} \frac{\mathrm{d}^{2} \boldsymbol{u}}{\mathrm{d} t^{2}} \delta \boldsymbol{\varphi}_{k} ; \\
& A_{I}=-\sum_{n=1}^{s_{2}} \int_{V_{n}} \rho_{n} \boldsymbol{f} \delta \boldsymbol{u} \mathrm{d} V+\sum_{n=1}^{s_{1}} \int_{V_{n}} \boldsymbol{f} \delta \boldsymbol{u} \mathrm{d} V+\sum_{n=1}^{N} F_{m} \delta \boldsymbol{u}_{n}+\sum_{k=1}^{n} m_{k} \delta \boldsymbol{\varphi}_{k} ;
\end{aligned}
$$


here, $\delta \varepsilon_{i j}, \delta \Delta l$-the variation of the deformations of distributed and elongated lumped elements; $\rho_{n}$-material density $n$-th concentrated element; $m_{k}-$ weight $\kappa^{\text {th }}$ rigid body; $\boldsymbol{u}_{1}, \boldsymbol{u}_{k}, \delta \mathbf{u}_{1}, \delta \boldsymbol{u}_{k}$-vectors of displacements of points of distributed elements and centers of mass of rigid bodies and their variations; $\varphi, \delta \varphi$-vectors of angular displacements of rigid bodies and their variations; $V_{n}, \Sigma_{n}$-volume and surface $n$-th distributed item; $I_{n}$-central inertia tensor $n$-th rigid body; $F_{m}, M_{k}$-the principal vector and principal moment of the forces, applied to $K$-hard body; $s_{1}$-otherwise deformability elements; $s_{2}-$ otherwise the wire deformability.

If the dissipative mechanical system consists of deformable bodies, then instead of (2a) the uniform equations of the movetion are used:

$$
\tilde{\mu}_{n} \frac{\partial^{2} u_{i}}{\partial x_{j} \partial x_{j}}+\left(\tilde{\lambda}_{n}+\tilde{\mu}_{n}\right) \frac{\partial^{2} u_{j}}{\partial x_{i} \partial x_{j}}-\rho_{n} \frac{\partial^{2} u_{i}}{\partial t^{2}}=0, \quad x \in V_{n}
$$

and boundary conditions

$$
\begin{gathered}
u_{i}=0 \quad \text { at } x \in \sum_{u}, \\
{\left[\tilde{\lambda}_{n} \frac{\partial u_{k}}{\partial x_{k}} \delta_{i j}+\tilde{\mu}_{n}\left(\frac{\partial u_{i}}{\partial x_{j}}+\frac{\partial u_{j}}{\partial x_{i}}\right)\right] v_{j}, \quad \text { at } x \in \Sigma_{p},}
\end{gathered}
$$

were $i, j, k=1,2,3 ; n=1,2,3, \cdots, N ; \rho_{n}$-material density $n^{\text {th }}$ volume; $v_{j}-$ components of the normal to the surface $\Sigma_{p} ; x=\left(x_{1}, x_{2}, x_{3}\right)$-radius vector of the point of the body; $u_{i}$-components of the displacement vector. At the volume boundaries $V_{n}$ we assume continuity of the displacement vector components, normal and tangent to the stress interface. We seek the solution of problem (2) and (3) in the form

$$
u_{i}\left(x_{1}, x_{2}, x_{3}, t\right)=U_{i}\left(x_{1}, x_{2}, x_{3}\right) \mathrm{e}^{-i \omega t},
$$

were $\omega=\omega_{R}+i \omega_{I}-$ complex natural frequency; $U_{i}-$ complex eigenmode of oscillations. Values $\omega$ and $U_{i}$ we define solving the problem on eigenvalues:

$$
\begin{array}{ll}
x \in V_{n}: & \bar{\mu}_{n}\left(\omega_{R}\right) \frac{\partial^{2} U_{i}}{\partial x_{j} \partial x_{j}}+\left(\bar{\lambda}_{n}\left(\omega_{R}\right)+\bar{\mu}_{n}\left(\omega_{R}\right)\right) \frac{\partial^{2} U_{j}}{\partial x_{i} \partial x_{j}}+\rho_{n} \omega^{2} U_{i}=0 ; \\
x \in \Sigma_{u}: & U_{i}=0 ; \\
x \in \Sigma_{p}: & {\left[\bar{\lambda}_{n}\left(\omega_{R}\right) \frac{\partial U_{k}}{\partial x_{k}} \delta_{i j}+\bar{\mu}_{n}\left(\omega_{R}\right)\left(\frac{\partial U_{i}}{\partial x_{j}}+\frac{\partial U_{j}}{\partial x_{i}}\right)\right] \boldsymbol{v}=0}
\end{array}
$$

We consider a construction with a finite number of degrees of freedom, consisting of a finite number of material points and absolutely rigid bodies, connected by massless viscoelastic elements. If the rheological characteristics of the elements are the same, then this system is a finite-dimensional model of a dissipative homogeneous system, under various rheological characteristics of the elements, the system under consideration simulates a dissipative inhomogeneous system with a finite number of degrees of freedom. If some elements of the mechanical system are deformed, then the designs represent a system with distributed parameters. For natural oscillations, it is necessary to determine the natu- 
ral frequencies and damping coefficients of the natural oscillations. The Lagrange equations for the system under consideration have the form:

$$
\sum_{k=1}^{n}\left(a_{j k} \ddot{q}_{k}+\tilde{c}_{j k} q_{k}\right)=0, \quad(j=1,2,3, \cdots, n)
$$

were $a_{j k}$-symmetric positive definite matrix of generalized masses; $q_{k}$-generalized coordinates; $n$-number of degrees of freedom; $\tilde{c}_{j k}-\mathrm{a}$ symmetric matrix whose elements are the operator:

$$
\tilde{c}_{j k} \varphi(t)=c_{j k}\left[\varphi(t)-\int_{0}^{t} R_{j k}(t-\tau) \varphi(\tau) \mathrm{d} \tau\right] .
$$

here $c_{j k}$-elements of a symmetric positive definite matrix of instantaneous generalized rigidities; $R_{j k}$-elements of a nonnegatively definite matrix of generalized relaxation kernels. For a homogeneous system (all elements are made of a single viscoelastic material and described by the same relaxation nuclei, but different instantaneous stiffnesses due to different sizes), all the relaxation nuclei (7) are the same. Consequently, the matrix of generalized operator stiffnesses is a positive definite real number matrix, multiplied by the operator scalar.

Thus, in normal coordinates $\theta_{\kappa}$ the elastic system (6) takes the form

$$
\theta_{\kappa}^{\prime \prime}+\Omega_{\kappa}^{2}\left[\theta_{\kappa}-\int_{0}^{t} R(t-\tau) \theta_{k}(\tau) \mathrm{d} \tau\right]=0,
$$

were, $\Omega_{\kappa}$-natural oscillation frequency of the elastic system. The system (8) splits into separate equations, that from the mechanical point vision, means the independence of the variation of various normal coordinates with free oscillations. For an inhomogeneous system (its deformable elements have different rheological characteristics, in particular, some of them may be elastic), the operator coefficients $\tilde{c}_{j k}$ in (6) is the sum of two matrices-the numerical and the operator matrix:

$$
\tilde{c}_{j k}=c_{j k}+\tilde{B}_{j k} ; \tilde{B}_{j k} \varphi=c_{j k}+\int_{0}^{t} R_{j k}(t-\tau) \varphi(\tau) \mathrm{d} \tau .
$$

Three symmetric matrices $a_{j k}, R_{j k}, c_{j k}$ can not, in the general case, be reduced to a diagonal form by a single no degenerate transformation; therefore, for a dissipative inhomogeneous system of Lagrange's equation in normal coordinates $\theta_{\kappa}$ does not decay, but takes the form:

$$
\theta_{\kappa}^{\prime \prime}+\Omega_{\kappa}^{2}\left[\theta_{\kappa}-\sum_{j=1}^{n} \int_{0}^{t} \Theta_{k j}(t-\tau) \theta_{k}(\tau) \mathrm{d} \tau\right]=0,
$$

were $\Theta_{k j}$-symmetric positive definite matrix of generalized relaxation kernels in the normal coordinates of the elastic problem. The difference between systems (8) and (9) can be interpreted as a fact of the mutual influence of the normal coordinates of a dissipative inhomogeneous system with free oscillations. This mutual influence, which, as a rule, is neglected, can be of fundamental importance. The problems of the natural oscillations of the system, described by Equations (6), can be solved in the following way. We replace the operators $c_{j k}$ in 
(6), by complex generalized rigidities: $\bar{c}_{j k}=c_{j k}\left[1-\Gamma_{j k}^{C}\left(\omega_{R}\right)-i \Gamma_{j k}^{S}\left(\omega_{R}\right)\right]$.

A particular solution of system (6) is sought in the form:

$$
q_{k}=U_{k} \mathrm{e}^{-i \omega t},
$$

were $\omega=\omega_{R}+i \omega_{I}$-desired complex natural frequency; $U_{k}$-components of the desired complex eigenforms. For the quantities $\omega$ and $U_{k}$ one can write a homogeneous linear algebraic system with a nonlinearly entering complex parameter. The characteristic equation of the eigenvalue problem has the form

$$
c_{j k}\left[1-\Gamma_{j k}^{C}\left(\omega_{R}\right)-i \Gamma_{j k}^{S}\left(\omega_{R}\right)\right]-\omega^{2} a_{j k}=0
$$

The roots of Equation (11) can be determined by the method of Mueller assuming the eigenfrequencies of the elastic problem as the initial approximation. The left-hand side of Equation (11) is calculated at each iteration by the method of Gauss with the separation of the principal element. Thus, to solve equation (11) we do not need to disclose the determinant on the left-hand side of.

As an example, consider a system with two degrees of freedom, consisting of two bodies of masses $m_{1}=1, m_{1}=1$, and three deformable elements with operator stiffnesses $\tilde{c}_{1}, \tilde{c}_{2}=\tilde{c}_{3}$ (Figure 1).

The dependence of the natural frequencies and damping factors on the instantaneous stiffness $c_{2}$ at fixed values $m_{1}, m_{1}, c_{1}$ and relaxation nucleus $R_{1}$, $R_{2}$. We consider two variants of the problem. For the first variant we have a homogeneous system $R_{1}=R_{2}=A \mathrm{e}^{-\beta t} / t^{1-\alpha}$, were $A=0.01$;

$\beta=1 ; \alpha=0.1 ; c_{1}=1$; instant stiffness $c_{2}=10^{-3}$ which considered by Koltunov M.A., Mayboroda V.P., Zubcheninov V.G. [4]. The results of the calculations are shown in Figure 1. Dependence of natural frequencies and damping factors on rigidity $c_{2}$ monotonic, and the character of the dependence is the same for frequencies and damping coefficients. In the second variant, a dissipative inho-

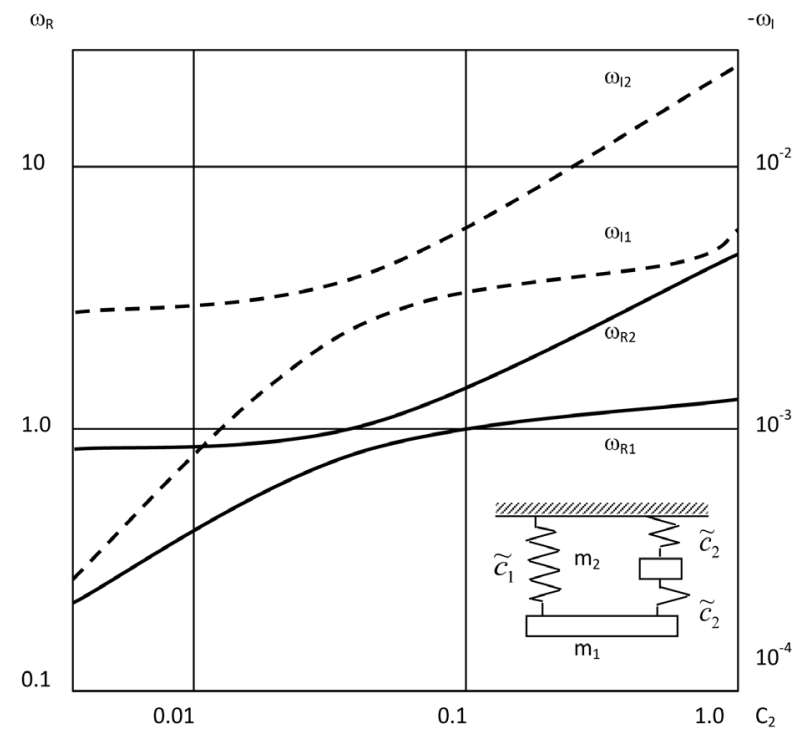

Figure 1. The change in complex frequencies as a function of $c_{2}$, for dissipative homogeneous mechanical systems. 
mogeneous system is considered: the first deformable element is elastic, $\left(R_{1}=0\right)$ the other parameters are the same as for the first option. The results of the calculations are shown in Figure 2. Dependence of natural frequencies $\omega_{R k}$ from $c_{2}$ the same as in the case of a homogeneous system, the corresponding curves coincide with an accuracy of up to $5 \%$. As for the coefficients of damping, their behavior varies radically: the dependence $\omega_{l k}$ from $c_{2}$ becomes no monotonic. Of particular interest is the minimum value of the damping coefficient for a fixed value $c_{2}$ damping factor:

$$
\delta_{\omega}=\min _{k}\left(-\omega_{I k}\right)
$$

Value $\delta$ determines the damping properties of the system as a whole. In the case of a homogeneous system, the quantity $\delta_{\omega}$ (we call it the global damping factor) is entirely determined by the imaginary part of the smallest modulo complex eigenfrequency. In the case of an inhomogeneous system, as the global damping factor as a function of the magnitude $c_{2}$ the imaginary parts of both the first and second eigenfrequencies appear. "Change of roles" occurs with a characteristic value of the value $c_{2}$, when the real parts of the first and second eigenfrequencies are closest. The global damping factor at the specified characteristic value $c_{2}$ has a pronounced maximum. This circumstance represents, in our opinion, a new mechanical effect, which can be formulated as follows: oscillations of the eigenmodes of a dissipative inhomogeneous viscoelastic system with close frequencies mutually cancel each other. Instant stiffness $c_{2}$ is a geometric parameter, determined by the dimensions of the element, and not by the physical properties of the material. The main feature of the observed effect is the qualitative dependence of the dissipative properties of the system on its geometric parameters.

Thus, the results obtained for the dissipative inhomogeneous viscoelastic design under consideration are completely consistent with the solutions of the problem of free damping oscillations and confirm the fact of a sharp increase in the intensity of dissipative processes when the fundamental frequencies approach

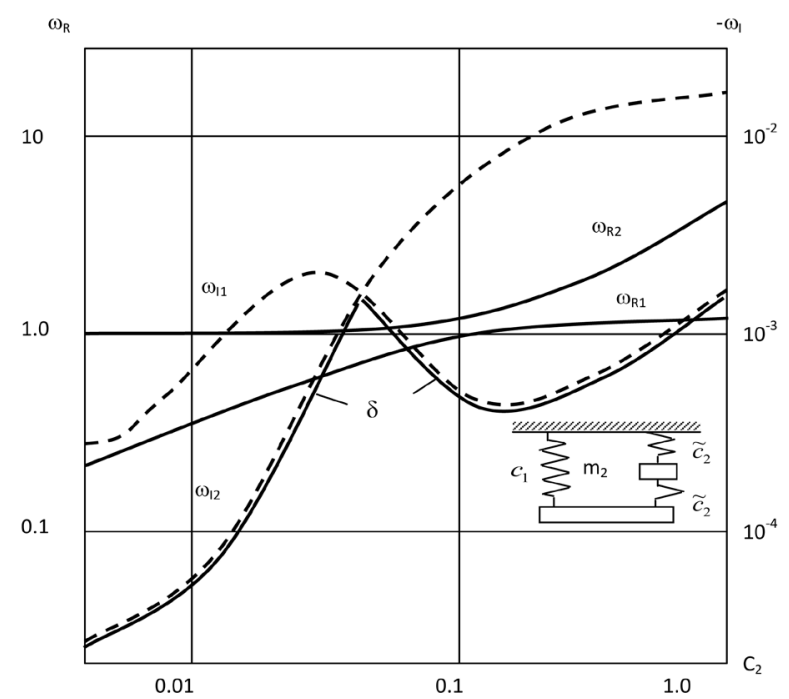

Figure 2. The dependence of complex frequencies on $c_{2}$. 
in inhomogeneous viscoelastic systems. In this case, the role of rheology reduces both to the damping of oscillations and to the mutually increasing interaction of oscillations and different modes, which substantially increases the dissipative properties of the system as a whole. This effect is called "the effect of Troyanovsky-Safarov".

The effect of interaction of various forms of motion of solid bodies has a principal perspective for the synthesis of dissipative heterogeneous engineering structures, construction products, damping compounds, materials and composites of various vibration protection systems and devices that are optimal for dissipative properties and material consumption.

Let us consider the natural oscillations of a system with two degrees of freedom (Figure 3). The following parameter values [2] are accepted:

$A=0.048 ; \beta=0.05 ; \alpha=0.1 ; c_{1}=1 ; M=1$, instant stiffness $C_{2}$ varies within $0.001 \div 1.5$, which was later contradicted by Bozorov M.B., Safarov I.I., Shokin Yu.I. [8].

Each motion of an inhomogeneous system is a superposition of interacting oscillations of several normal coordinates and this interaction of different normal coordinates, the most intense at close natural frequencies, leads to an intensification of the dissipative processes in the system. Let the dissipative mechanical system consist of layered bodies. As an example, consider a hollow cylinder of length $l$, consisting of coaxial viscoelastic layers. We introduce a cylindrical coordinate system: $r, \varphi, z$, which was later contradicted by Safarov I.I, Akhmedov M. Sh., Boltaev Z.I. [9]. Internal $r=R_{0}$ and the outer surface is free of stress $\sigma_{r r}=\sigma_{r \varphi}=\sigma_{r z}=0$; at the ends $z=0, z=l$ offsets $u_{z}=0$ and shearing stresses: $\sigma_{r z}=\sigma_{\varphi z}=0$; on the boundaries of layers: $r=r_{1} r_{2} \cdots r_{N-1}$ offsets: $u_{r}, u_{\varphi}, u_{z}$ and stresses $\sigma_{r r}, \sigma_{r \varphi}, \sigma_{r z}$ are continuous. As the required task variables, we take the $u_{r}, u_{\varphi}, u_{z}$ and stresses $\sigma_{r r}, \sigma_{r \varphi}, \sigma_{r z}$. Equations for these variables are obtained by eliminating from the Cauchy relations, Hooke's law and the equations of motion of strains and stresses $\sigma_{\varphi \varphi}, \sigma_{z z}, \sigma_{\varphi z}\left(r_{n-1}<r<r_{n}, n=1, \cdots, N\right)$, which was later contradicted by Safarov I.I, Akhmedov M. Sh., Boltaev Z.I. [10]:

$$
\begin{gathered}
\frac{\partial u_{r}}{\partial r}=-\frac{\bar{v}_{n}}{1-\bar{v}_{n}}\left(\frac{u_{r}}{r}+\frac{1}{r} \frac{\partial u_{\varphi}}{\partial \varphi}+\frac{\partial u_{z}}{\partial z}\right)+\frac{\left(1+\bar{v}_{n}\right)\left(1-2 \bar{v}_{n}\right)}{\bar{E}_{n}\left(1-\bar{v}_{n}\right)} \sigma_{r r} \\
\frac{\partial u_{\varphi}}{\partial r}=-\frac{1}{r} \frac{\partial u_{r}}{\partial \varphi}+\frac{u_{\varphi}}{r}+\frac{2\left(1+\bar{v}_{n}\right)}{\bar{E}_{n}} \sigma_{r \varphi} ; \\
\frac{\partial u_{z}}{\partial r}=-\frac{\partial u_{r}}{\partial z}+\frac{2\left(1+\bar{v}_{n}\right)}{\bar{E}_{n}} \sigma_{r z} ; \\
\frac{\partial \sigma_{r r}}{\partial r}=-\frac{1}{r} \frac{\partial \sigma_{r \varphi}}{\partial \varphi}-\frac{\partial \sigma_{r z}}{\partial z}-\frac{\sigma_{r r}-\sigma_{\varphi \varphi}}{r}+\rho_{n} \frac{\partial^{2} u_{r}}{\partial t^{2}} \\
\frac{\partial \sigma_{r \varphi}}{\partial r}=-\frac{1}{r} \frac{\partial \sigma_{\varphi \varphi}}{\partial \varphi}-\frac{\partial \sigma_{\varphi z}}{\partial z}-\frac{2}{r} \sigma_{r \varphi}+\rho_{n} \frac{\partial^{2} u_{\varphi}}{\partial t^{2}} ; \\
\frac{\partial \sigma_{r z}}{\partial r}=-\frac{1}{r} \frac{\partial \sigma_{r \varphi}}{\partial \varphi}-\frac{\partial \sigma_{z z}}{\partial z}-\frac{1}{r} \sigma_{r z}+\rho_{n} \frac{\partial^{2} u_{z}}{\partial t^{2}}
\end{gathered}
$$

where 


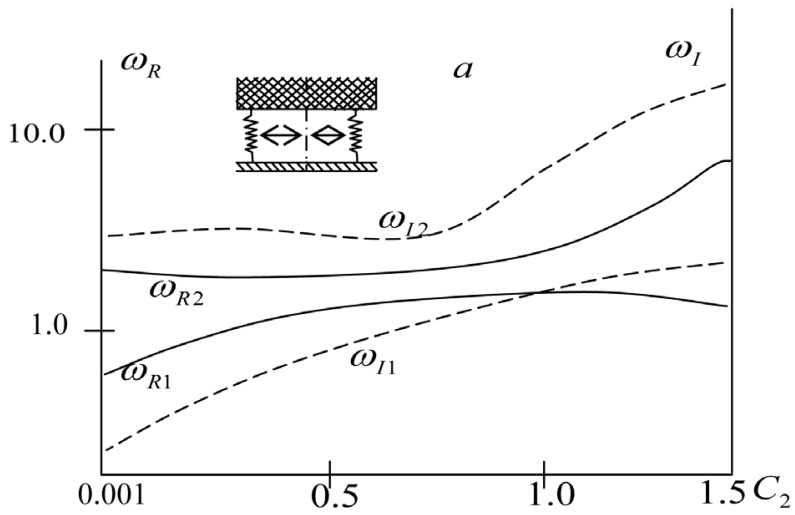

(a)

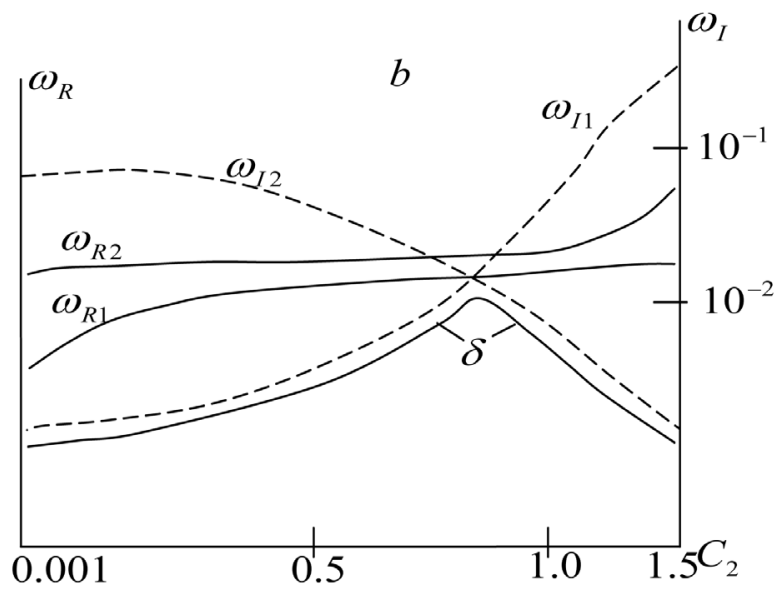

(b)

Figure 3. The dependence of complex frequencies on $C_{2}$; (a) Dissipatively homogeneous mechanical system; (b) Dissipatively homogeneous mechanical system.

$$
\begin{aligned}
\sigma_{\varphi \varphi} & =\frac{\bar{E}_{n}}{2\left(1+\bar{v}_{n}\right)}\left(\frac{u_{r}}{r}+\frac{1}{r} \frac{\partial u_{\varphi}}{\partial \varphi}+\bar{v}_{n} \frac{\partial u_{z}}{\partial z}\right)+\frac{\bar{v}_{n}}{1-\bar{v}_{n}} \sigma_{r r} ; \\
\sigma_{\varphi z} & =\frac{\bar{E}_{n}}{2\left(1+\tilde{v}_{n}\right)}\left(\frac{\partial u_{\varphi}}{\partial z}+\frac{1}{r} \frac{\partial u_{z}}{\partial \varphi}\right) ; \\
\sigma_{z z} & =\frac{\bar{E}_{n}}{1-\bar{v}_{n}^{2}}\left(\bar{v}_{n} \frac{u_{r}}{r}+\frac{\bar{v}_{n}}{r} \frac{\partial u_{\varphi}}{\partial \varphi}+\frac{\partial u_{z}}{\partial z}\right)+\frac{\bar{v}_{n}}{1-\bar{v}_{n}} \sigma_{r r} ; \\
\tilde{E}_{n} & =\frac{\bar{\mu}_{n}\left(3 \bar{\lambda}_{n}+2 \bar{\mu}_{n}\right)}{\bar{\mu}_{n}+\bar{\lambda}_{n}}, \bar{v}_{n}=\frac{\bar{\lambda}_{n}}{2\left(\bar{\mu}_{n}+\bar{\lambda}_{n}\right)} .
\end{aligned}
$$

The boundary conditions of the problem have the form:

$$
\begin{aligned}
& r=R_{0}, r=r_{n}: \quad \sigma_{r r}=\sigma_{r \varphi}=\sigma_{r z}=0 ; \\
& Z=0, z=l: \quad \sigma_{r z}=\sigma_{\varphi z}=0, u_{z}=0
\end{aligned}
$$

The problem (12)-(13) does not degenerate when $\tilde{V}_{n}=0.5$ (incompressible medium).

We seek the solution of the problem in the form: 


$$
\begin{aligned}
& \left(\begin{array}{l}
u_{r} \\
u_{\varphi} \\
u_{z}
\end{array}\right)=\left(\begin{array}{c}
U_{r} \\
i U_{\varphi} \\
i U_{z}
\end{array}\right) \mathrm{e}^{-i\left(\frac{k \pi}{l} z+\omega t+m \varphi\right),} \\
& \left(\begin{array}{c}
\sigma_{r r} \\
\sigma_{r \varphi} \\
\sigma_{r z}
\end{array}\right)=\left(\begin{array}{c}
\tau_{r r} \\
i \tau_{r \varphi} \\
i \tau_{r z}
\end{array}\right) \mathrm{e}^{-i\left(\frac{k \pi}{l} z+\omega t+m \varphi\right),}
\end{aligned}
$$

where $m, k$-positive integers; $U_{r}, U_{\varphi}, U_{z}, \tau_{r r}, \tau_{r \varphi}, \tau_{r z}$-complex amplitudes depending on the radius $r$. The problem reduces to finding the eigenvalues for a system of six ordinary differential equations with respect to complex amplitudes $\left(r_{n-1}<r<r_{n}, n=1, \cdots, N\right)$ :

$$
\begin{aligned}
\frac{\mathrm{d} U_{r}}{\mathrm{~d} r} & =-\frac{\bar{v}_{n}}{1-\bar{v}_{n}}\left(\frac{U_{r}}{r}-i \frac{m}{r} U_{\varphi}-i \frac{k \pi}{l} U_{z}\right)+\frac{\left(1+\bar{v}_{n}\right)\left(1-2 \bar{v}_{n}\right)}{\bar{E}_{n}\left(1-\bar{v}_{n}\right)} \tau_{r r} ; \\
\frac{\mathrm{d} U_{\varphi}}{\mathrm{d} r} & =i \frac{m}{r} U_{r}+\frac{U_{\varphi}}{r}+\frac{2\left(1+\bar{v}_{n}\right)}{\bar{E}_{n}} \tau_{r \varphi} ; \\
\frac{\mathrm{d} U_{z}}{\mathrm{~d} r} & =i \frac{k \pi}{l} U_{r}+\frac{2\left(1+\bar{v}_{n}\right)}{\bar{E}_{n}} \tau_{r z} ; \\
\frac{\mathrm{d} \tau_{r r}}{\mathrm{~d} r} & =i \frac{m}{r} \tau_{r \varphi}+i \frac{k \pi}{l} \tau_{r z}-\frac{\tau_{r r}-\tau_{r \varphi}}{r}-\rho_{n} \omega^{2} U_{r} ; \\
\frac{\mathrm{d} \tau_{r \varphi}}{\mathrm{d} r} & =i \frac{m}{r} \tau_{\varphi \varphi}+i \frac{k \pi}{l} \tau_{\varphi z}-\frac{2 \tau_{r \varphi}}{r}-\rho_{n} \omega^{2} U_{\varphi} ; \\
\frac{\mathrm{d} \tau_{r z}}{\mathrm{~d} r} & =i \frac{m}{r} \tau_{r \varphi}+i \frac{k \pi}{l} \tau_{r z}-\frac{\tau_{r z}}{r}-\rho_{n} \omega^{2} U_{z} .
\end{aligned}
$$

here

$$
\begin{aligned}
\tau_{\varphi \varphi} & =\frac{\bar{E}_{n}}{2\left(1+\bar{v}_{n}\right)}\left(\frac{U_{r}}{r}-i \frac{m}{r} U_{\varphi}-i \bar{v}_{n} \frac{k \pi}{l} U_{r}\right)+\frac{\bar{v}_{n}}{1-\bar{v}_{n}} \tau_{r r} ; \\
\tau_{\varphi z} & =-\frac{i \bar{E}_{n}}{2\left(1+\bar{v}_{n}\right)}\left(\frac{k \pi}{l} U_{\varphi}+\frac{m}{r} U_{z}\right) ; \\
\tau_{z z} & =\frac{\bar{E}_{n}}{1-\bar{v}_{n}^{2}}\left(\bar{v}_{n} \frac{U_{r}}{r}-i \frac{\bar{v}_{n} m}{r} U_{\varphi}-i \frac{k \pi}{l} U_{z}\right)+\frac{\bar{v}_{n}}{1-\bar{v}_{n}} \tau_{r r} ;
\end{aligned}
$$

when

$$
r=R_{0}, r=r_{n}: \tau_{r r}=\tau_{r \varphi}=\tau_{r z}=0 ; z=0, z=l: \tau_{r z}=\tau_{\varphi z}=0, U_{z}=0 .
$$

The characteristic equation of the problem is constructed by the method of orthogonal sweep, the roots of the characteristic equation are determined by the Muller method. Figure 4 shows the results of calculations for the case of plane oscillations $(m=2, k=0)$ cylinder, consisting of an outer thin elastic layer $\left(r_{1}=1.5 ; r_{2}=1.7\right.$ and an internal incompressible viscoelastic layer). Inner radius $R_{0}$, which varied from 0.6 to 1.3 . The following parameter values are accepted: $E_{1}=10^{-4} v_{1}=0 / 5, R_{\mu 1}=A \mathrm{e}^{-\beta t} / t^{1-\alpha}, A=0.01 ; \quad \beta=1 ; \alpha=0.1 ; \quad E_{2}=1 ; \quad v_{2}=0.3$; $R_{2}=0$. Figure 4 shows the dependences of the real and imaginary parts of the first two natural frequencies on the inner radius $R_{0}$. The above dependences confirm the effect found in the analysis of the natural oscillations of a system 


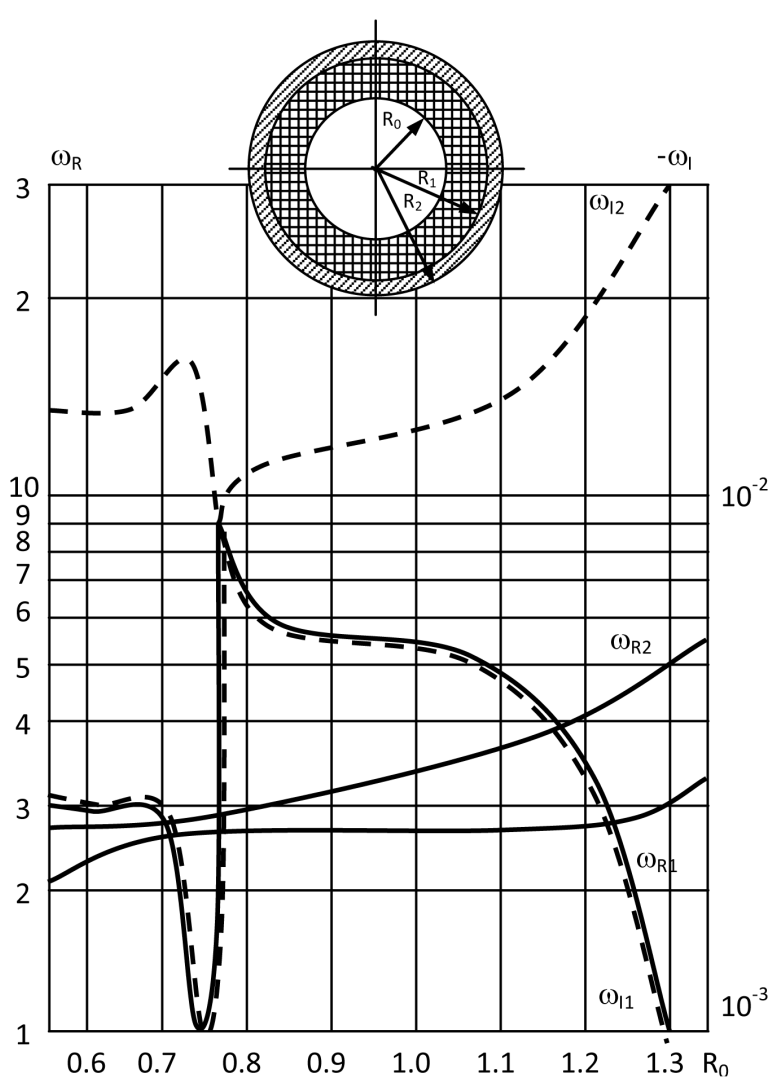

Figure 4. The change in complex frequencies as a function of $c_{2}$, for dissipative inhomogeneous mechanical systems.

with a finite number of degrees of freedom.

\section{Forced Oscillations of Dissipative Mechanical Systems}

For a system with a finite number of degrees of freedom, the variational problem (2a) reduces to a system of linear Lagrange equations of the second kind with complex generalized rigidity:

$$
\sum_{k=1}^{N}\left(a_{j k} \ddot{q}_{k}+\tilde{c}_{j k} q_{k}\right)=f_{j} \mathrm{e}^{-i \lambda t},(j=1,2,3, \cdots, N)
$$

where

$$
\tilde{c}_{j k} \varphi(t)=c_{j k}\left[\varphi(t)-\int_{-\infty}^{t} R_{j k}(t-\tau) \varphi(\tau) \mathrm{d} \tau\right],
$$

$a_{i j}$-components of the real symmetric matrix of generalized masses; $q_{k}$-complex generalized coordinates; $f_{j}$-complex amplitudes of generalized forces; $\lambda$-actual frequency of external influences. The solution of the problem of forced oscillations of system (16) is sought in the form:

$$
q_{j}=A_{j} \mathrm{e}^{-i \lambda t},
$$

where $A_{j}$ desired complex amplitudes. The problem of forced oscillations, reduces to solving an inhomogeneous algebraic system: 


$$
\sum_{j=1}^{N}\left(c_{l k}(\lambda)-\lambda^{2} a_{j k}\right) A_{k}=f_{j},
$$

the solution, which is carried out in the Gauss method. In the presence of a medium of deformable elements of a system of at least one with a distributed mass, the number of its degrees of freedom becomes infinite (countable). In this paper we propose two approaches to solving an infinite-dimensional version of the variational problem (2a). The first approach reduces to constructing an infinite system of Lagrange equations of the second kind, which after truncation turns into a finite system of the form (16). As the generalized coordinates, the components of the displacements of the centers of mass of rigid bodies, and their small angles of rotation with respect to the coordinate axes (or principal directions of inertia) are taken, as well as, the components of the expansion of the displacements of the massive deformable elements from their elastic self-oscillations (here we mean the eigenmodes of the vibrations of an elastic isolated deformable element). In this case the variational problem (2a) reduces to a system of partial differential equations, which has been widely studied [10]:

$$
\sum_{k=1}^{s_{2}} L_{j k} W_{j} \pm \rho_{j} \frac{\partial^{2} W_{j}}{\partial t^{2}}=\rho f_{j} \mathrm{e}^{-i \lambda t},
$$

where $W_{j}$-components of the displacement vector of points $j^{\text {th }}$ massive of the deformable element; $\rho_{j}$-its linear density, depending on the coordinates; $f_{j}-$ components of complex amplitudes of mass forces, including portable inertia forces for a given vibration of a rigid base; $L_{j k}$-linear combinations of differentiation operators with respect to the spatial coordinate of the $\mathrm{k}^{\text {th }}$ rod with complex coefficients. These coefficients in the case of natural oscillations depend on the real parts of the desired complex eigenfrequencies, and for the steadystate ones, on the actual frequency of the external action. The system (19) does not include masses and moments of inertia of absolutely rigid bodies and complex stiffnesses of massless deformable elements of zero volume. These parameters in the approach are taken into account, both in the boundary conditions and in the internal (depending on the location of the attached masses) points of the $j^{\text {th }}$ rod. As shearing forces and bending moments, the elastic forces of concentrated deformed elements, forces and moments of inertia forces (relative portable ones) applied to the absolute rigid body of the system will enter into the specified boundary conditions. The boundary conditions, for the system (19), in the general case are not written out in view of the extreme variety. Let us determine the dependence of the resonance amplitudes $A_{j k}\left(j^{\text {th }}\right.$ number of the generalized coordinate, $\mathrm{k}$ is the number of the resonant frequency ) (18) from the system parameters. The algorithm for constructing this dependence includes the construction of amplitude-frequency characteristics, for each generalized coordinate $q_{j}$ and finding the maximums $A_{j k}$ for each $\lambda_{j}$. To find the maxima and minima on the resonance curve, the Mueller method is applied, which has been widely studied [10]. The amplitude-frequency response, this curve, is described by equation $A_{j}(\lambda)=\left|q_{j}(\lambda)\right|$. Its maxima and minima satisfy equation 


$$
\frac{\partial A_{j}(\lambda)}{\partial \lambda}=0
$$

It is necessary to have the roots of this equation (by the Muller method).

To do this, we must calculate the left-hand sides of (20) for given values $\lambda=\lambda_{1}, \lambda_{2}, \cdots, \lambda_{n}$. Having chosen $\Delta \lambda=10^{-4}-10^{-6}$, we define the derivatives by the formula:

$$
\frac{\partial A_{j}(\lambda)}{\partial \lambda}=\frac{A_{j}\left(\lambda_{j}+\Delta \lambda\right)-A_{j}\left(\lambda_{j}-\Delta \lambda\right)}{2 \Delta \lambda}=0 .
$$

The first root is known $\lambda=0$, so you need to start looking for the second one. As an example, we consider a system with two degrees of freedom (Figure 3 ), which has been widely studied [11]. The values are accepted, the other parameters coincide with those adopted in the second task. Two variants of mechanical systems are considered. The results of the calculation of the first variant are shown in Figure 5(a). Addiction $A_{j k}(j=1,2 ; k=1,2)$ of the parameter $c_{2}$ monotonous. In the second variant, the first deformable element is elastic: $R_{1}=0$, the other parameters are the same as those used above. The results of the calculation are shown in Figure 5(b), according to which when the natural frequencies approach the corresponding amplitudes $A_{11}$ and $A_{12}$ becomes equal. Addiction $A_{j k}$ from $c_{2}$ no monotonic. The damping properties of the system as a whole for forced oscillations are determined by the maximum resonance amplitude (we call it the global resonant amplitude). The intensity of dissipative processes in the system is higher the lower the global resonant amplitude (GRA): $\delta_{A}=\max _{\lambda}\left(A_{j k}\right)$.

In a dissipative homogeneous system, the role of the global resonant amplitude is fulfilled for all values of the parameter by the first resonance amplitude. In a dissipative inhomogeneous system, both the first and second resonant amplitudes act as the GRA in terms of the magnitude of the parameter. "Change of roles" also occurs in the case of a global damping factor $\delta_{A}$ with a characteristic value of the parameter at which the real parts of the natural frequencies are closest.

At this value of the parameter, the global resonant amplitude (GRA) is minimal and, consequently, the dissipative processes in the system proceed most intensively, and the global damping coefficient has a pronounced maximum. This effect is a continuation of the effect of Troyanovsky-Safarov. To clarify the physical nature of the detected effect, we write the equations of motion of a system with $\mathrm{n}$ degrees of freedom. In the case of a homogeneous system, all the relaxation nuclei $R_{i j}$ is the same: $R_{i j}=R$, since the matrix of generalized complex rigidity $c_{i j}$ is a positive definite real matrix $c_{i j}$, multiplied by a complex scalar:

$$
\bar{c}_{j k}=c_{j k}\left[1-\Gamma_{j k}^{C}\left(\omega_{R}\right)-i \Gamma_{j k}^{S}\left(\omega_{R}\right)\right] .
$$

In the normal coordinates of the viscoelastic problem, the system (16) takes the form:

$$
\ddot{\theta}_{n}+\Omega_{n}^{2} \theta_{n}\left(1-\Gamma^{c}-i \Gamma^{s}\right)=\Psi_{n}
$$




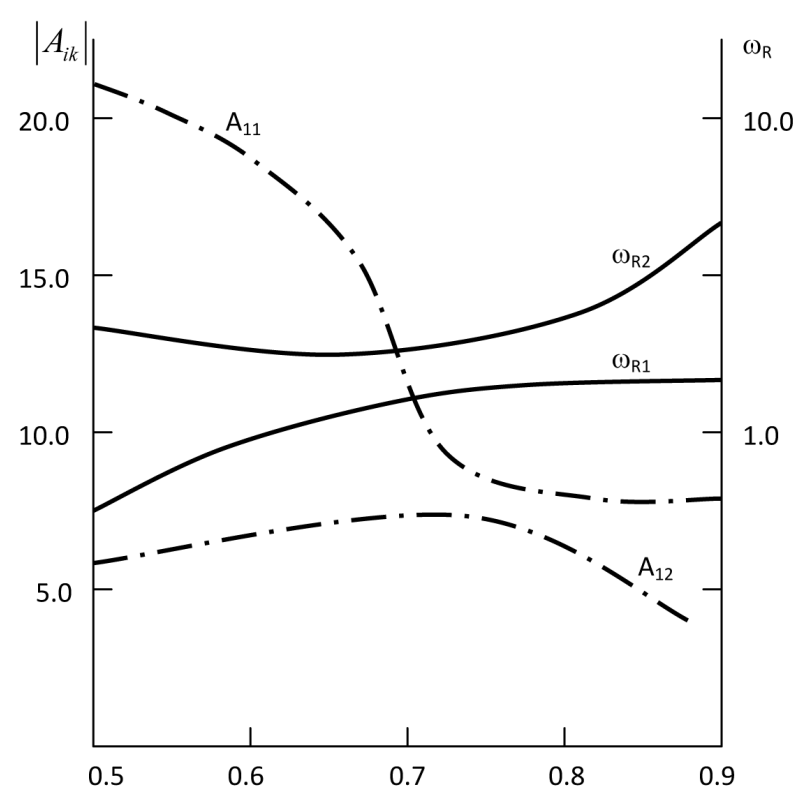

(a)

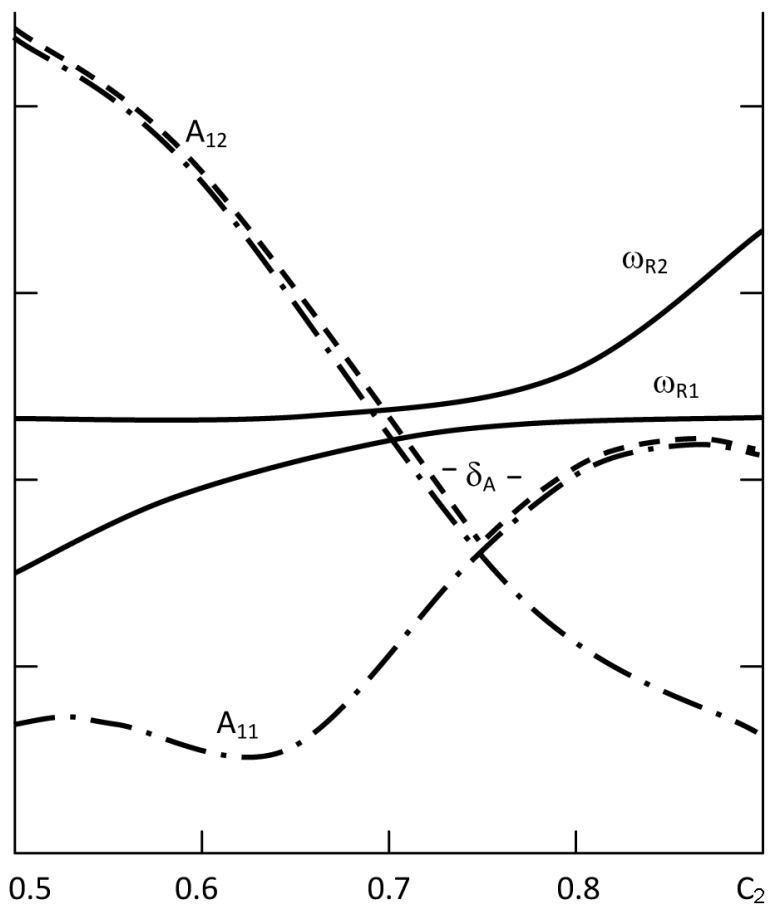

(b)

Figure 5. Dependence of the resonance amplitude on $c_{2}$; (a) Dissipatively homogeneous mechanical system; (b) Dissipatively homogeneous mechanical system.

where $\Omega$-complex own frequency of elastic system; $\Psi_{n}$-generalized force corresponding to $n^{\text {th }}$ normal coordinate. The system (21) is divided into $\mathrm{n}$ separate equations. This means that, the motion of a mechanical viscoelastic system is a superposition of independent normal vibrations decay, and the forced ones have a finite resonant amplitude. The main property of conservative systems- 
the possibility of excitation of the vibration of one normal coordinate without excitation of the others-is completely preserved in the case of a homogeneous viscoelastic system. Therefore, in the case of an inhomogeneous system, the Lagrange equation in normal cords of an elastic system has the form

$$
\ddot{\theta}_{n}+\Omega_{n}^{2} \theta_{n}-\Omega_{n}^{2} \sum_{j=1}^{N}\left(\theta_{n j}^{c}+\theta_{n j}^{s}\right) \theta_{j}=\Psi_{k}
$$

where $\theta_{n j}^{c}, \theta_{n j}^{s}$-non-negative definition of a real matrix. The system (22) consists of related equations. This mechanical coupling means that it is impossible to excite the oscillation of an individual normal coordinate. This effect has been widely studied [12] [13] [14]. Each motion of an inhomogeneous system is a superposition of interacting oscillations of several normal coordinates, and this interaction of different normal coordinates, the most intense at close natural frequencies, leads to an intensification of the dissipative processes in the system.

\section{References}

[1] Safarov, I.I. (1992) Vibrations of a Wave in Dissipatively Inhomogeneous Media and Structures. Tashkent: Fan, $252 \mathrm{p}$.

[2] Bozorov, M.B., Safarov, I.I. and Shokin, Yu.I. (1996) Numerical Simulation of Oscillations of Dissipatively Homogeneous and Inhomogeneous Mechanical Systems. Sibirian Branch of the Russian Akademy of Science, Novosibirsk, 189 p.

[3] Safarov, I.I., Teshaev, M.Kh. and Majidov, M. (2012) Damping of Oscillations of Dissipative-Inhomogeneous Mechanical Systems. Foundations, Concepts, Methods. LAP, Lambert Academic Publishing (Germany), 8 p.

[4] Koltunov, M.A., Mayboroda, V.P. and Zubcheninov, V.G. (1983) Strength Calculations of Products Made of Polymer Materials. Mashinostroenie, Moscow, 239 p.

[5] Koltunov, M.A. (1976) Creep and Relaxation. Higher Shkola, Moscow, 277 p.

[6] Leibenzon, L.S. (1943) Variational Methods for Solving Problems in the Theory of Elasticity. Gostekhizdat, Moscow, 286 p.

[7] Mayboroda, V.P., Troyanovsky, I.E. and Safarov, I.I. (1983) Free and Forced Oscillations of Systems of Solids on Inhomogeneous Viscoelastic Shock Absorbers. Journal of the USSR Academy of Sciences, 3, 71-77.

[8] Safarov, I.I., Teshaev, M.Kh. and Madjidov, M. (2014) Natural Oscillations of Viscoelastic Lamellar Mechanical Systems with Point Communications. Applied Mathematics, 5, 3018-3025. https://doi.org/10.4236/am.2014.519289

[9] Safarov, I.I., Akhmedov, M.Sh. and Boltaev, Z.I. (2014) Loose Waves in Viscoelastic Cylindrical Wave Guide with Radial Crack. Applied Mathematics, 6, 214-225. https://doi.org/10.4236/am.2014.521329

[10] Safarov, I.I., Akhmedov, M.Sh. and Boltaev, Z.I. (2015) Setting the Linear Oscillations of Structural Heterogeneity. Viscoelastic Lamellar Systems with Point Relations. Applied Mathematics, 6, 225-234. https://doi.org/10.4236/am.2015.62022

[11] Safarov, I.I., Akhmedov, M.Sh. and Boltaev, Z.I. (2015) Natural Oscillations of Cylindrical Bodies with External Friction on the Boundary. Applied Mathematics, 6, 629-645. https://doi.org/10.4236/am.2015.63057

[12] Safarov, I.I., Boltaev, Z.I. and Umarov, A.O. (2013) Forced Oscillations of Cylindrical Bodies with External Friction at the Boundary. Interuniversity Collection of Scientific Papers "Problems of Mechanics and Management. Nonlinear Dynamic 
Systems. Permian, 45, 114-122.

[13] Safarov, I.I., Teshaev, M.K.H. and Boltaev, Z.I. (2016) Waves in a Cylindrical Shell with a Viscous Liquid. Vestnik of Perm University. Mathematics. Mechanics. Computer Science, Perm, 3, 82-93.

[14] Safarov, I.I., Boltaev, Z.I. and Axmedov, M.Sh. (2016) Ford Hormonal Vibrations in a Visocoelastic Layered Bodies Lying Deformable Nalf-Plane. International Journal of Applied Research, 2, 138-147.

Submit or recommend next manuscript to SCIRP and we will provide best service for you:

Accepting pre-submission inquiries through Email, Facebook, LinkedIn, Twitter, etc. A wide selection of journals (inclusive of 9 subjects, more than 200 journals)

Providing 24-hour high-quality service

User-friendly online submission system

Fair and swift peer-review system

Efficient typesetting and proofreading procedure

Display of the result of downloads and visits, as well as the number of cited articles

Maximum dissemination of your research work

Submit your manuscript at: http://papersubmission.scirp.org/

Or contact am@scirp.org 